\title{
Young Adulthood and Unique Challenges in Living with Type 1 Diabetes
}

\author{
Michael James Curtis Jr ${ }^{\mathrm{a}}$, Samereh Abdoli ${ }^{\mathrm{b}}$, Joanne Hall ${ }^{\mathrm{c}}$
}

Type 1 Diabetes Mellitus (Type I DM) presents young adults with unique challenges in managing diabetes while they navigate transition from adolescence to adulthood. This article presents a narrative review of literature pertaining to challenges surrounding diabetes care in young adults with Type I DM. Methods: A literature search was conducted in CINAHL, PubMed and Web of Science for studies related to diabetes care in young adults with Type I DM. The search was completed using various combinations of diabetes, Type I DM, diabetes care/management, challenges, and barriers in English. Results: Based on the study findings the most challenges experienced by young adults were associated to "psychosocial challenges", "life style change", "lack of supportive network", and "delivery of diabetes care." However, diabetes education particularly related to life style is necessary; individualized psychosocial and behavioral interventions should be considered as an essential part of diabetes care systems. A more supportive health care system and community can impact positively on quality of life and diabetes care in young adults living with Type I DM.

Key words: Type 1 Diabetes Mellitus, Transition to adulthood, Diabetes care, Young adults

\section{Introduction:}

Health disparity is a difference in health outcomes, adversely affecting groups who have experienced barriers to health, linked to characteristics including poverty, race/ethnicity, color, gender, geographic environment, interpersonal environment, and developmental transitions. The goal of Healthy People 2020 is to achieve health equity, eliminate disparities, and improve the health of all groups (HHS 2014). There has been a steady increase in the awareness, measurement, and documentation of disparate health care trends across the United States. However, the reduction or elimination of most health disparities has remained elusive to providers, researchers, and policymakers (White, Beech et al. 2009).

Diabetes care is an important area of national focus as efforts continue toward eliminating health disparities and improving the overall health of all Americans. Disparities in both the processes of diabetes care and diabetes health outcomes persist across the country (White, Beech et al. 2009), specifically in adults in the period defined as emerging adulthood (18-25 years old) (Borak, Salipante-Zaidel et al. 2012).

Emerging adulthood (young adulthood) is a developmental period characterized by exploration, experimentation, and risk-taking (Pyatak 2010). Emerging adulthood represents a time of delaying permanent adult commitments and of exploring identities and life alternatives (Luyckx, Seiffge-Krenke et al. 2008). Emerging adults with diabetes face unique challenges in managing a complex illness requiring a high degree of predictability and routinization while carrying out the exploration, experimentation, and risk taking that is normative in this developmental period. They are likely to be experimenting with risky behaviors, uprooting their lives, and enjoying a period of relative freedom from responsibility, which can include avoiding the responsibility of diabetes management (Pyatak 2010).

Emerging adults with Type I DM, face more challenges managing their illness and navigating the life transitions of adulthood (Melton and Johnson, 2015). This places them at an increased risk for loss to follow-up and adverse health outcomes, including poor glycemic control, emergence of chronic diabetes complications, and premature mortality (Petitti, Klingensmith et al. 2009; Peters, Laffel et al. 2011; Garvey, Wolpert et al. 2012; Lotstein, Seid et al. 2013; Gill, Nayak et al. 2014). Young adults experience increased mean blood glucose level (Majumder, Cogen, et al 2016). Ketoacidosis is the most common complication among the young with uncontrolled diabetes arriving in the emergency department in the U.S. (Amaize and Mistry 2012). Hypoglycemia is also a common acute complication in this group (Feltbower, Bodansky, et al 2008). This population also experience higher rates of long term complications such as hypertension, retinopathy and neuropathy (Lin Perri, Gallaaagher, et al 2014). Diminished psychological wellbeing during emerging adulthood is an important concern in living with Type I DM. Thus, there has been an increase in diabetes mellitus hospitalizations, ultimately, causing a higher risk of mortality due to the hardship of during the transition to adulthood (Garvey \& Wolpert, 2011; Garvey, Markowitz \& Laffel, 2012). The purpose of this article is to provide a current, comprehensive review of the challenges young adults with Type I DM encounter during the early adulthood.

\section{Methods:}

A literature review was conducted, and articles were obtained through Cumulative Index of Nursing and Allied Health Literature (CINAHL), PubMed and Web of Science for studies relating to management of diabetes in young adults with Type I DM. Articles were chosen based on relativity to the concept, "challenges of young adults with diabetes self-

a. Nursing student, Honors program, University of Tennessee, College of Nursing, Knoxville, TN, 37996

b. PhD, RN. Assistant Professor, University of Tennesssee, College of Nursing, Knoxville, TN,37996

c. PhD, RN. Professor, University of Tennessee, Knoxville, TN, 37996

Correspondence: Dr. Samereh Abdoli, Phone: 865-974-7126, Email: sabdoli@utk.edu 
care." Keywords were used to narrow the results, which include, "challenges," "barriers," "Type I DM," "diabetes care/management," and "diabetes." Nineteen articles were chosen to be incorporate within this review. Within these articles, commons themes emerged, which will support the foundation of this review- "psychosocial challenges," "lifestyle changes," "lack of supportive networks," and "delivery of diabetes care."

\section{Results:}

The literature review highlighted major challenges that young adults with Type I DM may experience during transition to adulthood. These challenges included "psychosocial challenges", "life style change", "lack of supportive network", and "delivery of diabetes care." Each of these will be discussed with supportive evidence from literature.

\section{Psychosocial Challenges}

Psychosocial challenges occur more often in those with diabetes compared with those without diabetes, occurring more commonly during emerging adulthood than during other stages of life (Peters, Laffel et al. 2011). Approximately onethird of young adults with Type I DM experience psychological distress, which is associated with poor glycemic control. Fear of complications from diabetes, feeling different from peers, and frustrations with self-care management obstacles result in feelings of isolation, worry, and hopelessness. The emotional impact of diabetes was described as profound and something participants often cannot fully handle (Melton and Johnson 2015). Psychological distress is related to frequency of hypoglycemic episodes and method of insulin administration, with significantly greater distress being observed in those using multiple daily injection (MDI) (Kipps, Bahu et al. 2002).

Young adults are disproportionately more sensitized to other people's perceptions about their illness, Type I DM. This stemmed from past experience of being a sick/or disabled person (Abdoli, Mardanian, et al 2013, Weiler and Crist 2009), being considered unmarriageable (Kesavadev, Sadikot et al. 2014, Singh, Cinnirella et al. 2015, Abdoli, Mardanian, et al 2013 ), potentially feeling that one is a frustrating burden that the family has to carry (Maslakpak, Anoosheh et al. 2010, Kesavadev, Sadikot et al. 2014), or being labeled a drug abuser because of the misunderstanding of the injection of insulin as addiction. (Browne, Ventura et al. 2014, Pyatak 2011, Buchbinder, Detzer et al. 2005).

Emerging adults with diabetes are stigmatized due to the peers misunderstanding the illness, such as confusing medical supplies with that is drug abuse or implications of death. This leads to intentional privacy of diabetic care, and, in many case, privacy will lead to inconsistency with care (Pytak 2011). In many ways, emerging adults with Type I DM are made to feel "different" or "other." This results in feeling deprived of a normal life. Therefore, young adults with Type I DM try to present themselves as "normal" through concealing their illness to prevent diabetes-related stigma. They seek a safe and private place for testing their blood glucose (Pyatak 2011). Unfortunately, they often resort to unsanitary places such as toilets, and sometimes use unsanitary methods of selfcare. Young adults with Type I DM may deliberately ignore their diabetes symptoms and care, or delay it, to appear "normal" and to escape stigma. They also may limit their social interactions to avoid diabetes disclosure and escape similarly stigmatizing conversations (DoostiIrani, Abdoli et al. 2013). Fear of complications from diabetes, feeling different from peers, and frustrations with self- care management obstacles resulted in feelings of isolation, worry, and hopelessness. (Melton \& Johnson 2015).

Young adults with Type I DM depression experience more depression than those without diabetes, which is associated with adverse implications for self-care and $\mathrm{Hb}$ A1c levels (Johnson, Eiser et al. 2013). Recent research also indicates higher prevalence rates of eating disorders among young people with Type I DM, as compared to their peers without diabetes. Having diabetes as a young person does not protect one from the other common problems that may emerge in adolescence. However, these problems are highly complicated when they occur in those with the increased pathology of Type I DM. For example, eating disorders and disordered eating behaviors - especially insulin omission are linked to diabetes-related complications such as poorer glycemic control and pose serious risks for increased morbidity and mortality in this vulnerable population (Hanlan, Griffith et al. 2015).

Although identity development begins in adolescence, most of identity exploration and establishment takes place during emerging adulthood. Identity exploration is lower in emerging adults with diabetes than in comparison peers. Emerging adults with diabetes may have more difficulties establishing an independent identity than their peers. Greater likelihood of continuing parental involvement and oversight of the young person's diabetes self-care may be disruptive to identity development and sociocultural aspects of life, resulting in a lower sense of life purpose and/or life satisfaction during early emerging adulthood (Luyckx, Seiffge-Krenke et al. 2008).

Young adults with Type I DM have to reconcile the demands of their illness with the challenges of identity development (Abdoli, Ashktorab et al. 2014). Moreover, Type I DM negatively impacts young adults' identity, selfperceptions and social connections (Melton and Johnson 2015). They may experience a shift in identity and selfconcept upon their diagnosis of diabetes, coming to see themselves as "different," or "not normal," (Dovey-Pearce, Doherty et al. 2007). They fear being labeled as "a diabetic" and social rejection because of this label (Abdoli, Ashktorab et al. 2014). Thus, the characteristic of being diabetic becomes integrated into their identity in an intrusive, permeating way ("I am a diabetic") instead of in a way that preserves the core of the person, not as "diabetic" ("I am a person with diabetes"). Having an illness centered identity is related to poor diabetes metabolic control and poor physical and psychosocial well-being (Luyckx, Rassart et al. 2016, Abdoli, Ashktorab, etal 2014).

\section{Life-Style Change:}

Emerging young adults are expected to become more self-responsible about diabetes care with its constant daily requirements during their transition to adulthood. All of diabetes management activities are likely to have been monitored at least partially by parents during adolescence. But in the emerging adulthood period, they experience loss of 
parental supervision of diabetes care, and the challenges of work and/or school often take precedence over diabetes care. This may reduce their attendance at diabetes medical visits and may increase the risk of hypoglycemia and severe hyperglycemia or DKA (Peters, Laffel et al. 2011).

Emerging adults with Type I DM may encounter the challenges of "managing new schedules, changes in diet and physical activity, evolving relationships..., and peer pressure to engage in risky behavior" (Monaghan, Helgeson, \& Wiebe, 2015). Moreover, emerging adulthood marks social changes, physical growth, and intellectual transitions that are occurring at the same time. The developmental challenges of emergent sexual feelings, dating, maintaining friendships, and reproductive-related needs may be troublesome for young adults already overwhelmed by the demands of their diabetes self-care and the avoidance of stigmatization. Management of diabetes may be perceived as burdensome, interfering with the social aspects of their lives. They may not desire to allot time to diabetes management, such as checking glucose levels, eating nutritious meals, and injecting insulin before or after a meal, because it interrupts their time with social groups, academic schedule, or engagement in activities (Hanna 2012). The fear to interrupt the norm stems from the regard to disrupt the normal progression of activities causing unwanted attention and feeling of isolation due to methods to managing diabetes (Pytak 2011).

Although emerging adults have more freedom from parental supervision, they still experience limited control of their new schedules, routines, and lifestyles, often resulting in fewer appointments to health care professionals and unsuccessful management of hyperglycemic readings. The lack of scheduling will result in unpreparedness for unexpected events, such as hypoglycemia or diabetic ketoacidosis, and resolutions may come too late before damage has occurred (Pytak, 2011).

Emerging adults may indulge in consumption of alcohol or illegal drugs and engage in diet restrictions, self-induced vomiting, and skipping meals that may contribute to interference of glycemic control. The lack of a sufficient diet, insufficient monitoring of blood glucose due to poorly managed schedules, and lack of support from campus medical staff often leads to worsening glycemic control and quality of life (Monaghan et al., 2015; Garvey \& Wolpert, 2011; Hynes, Byrne, Casey, Dinneen, \& O'Hara, 2015). Diabetes management is not a priority to emerging adults during the time of great self-exploration and risk-taking. The lack of knowledge can lead to misinterpretation of glucose readings, misuse of diabetic materials, or non-compliance with the medical regime (Pytak 2011). Low competency and selfefficacy can influence the diabetic management, causing less acceptance of the illness and less glycemic control (Scholes, Mandleco et al. 2012).

\section{Lack of Supportive networks:}

There are links of diabetes management and parental/peer relationships that impact the emerging adultcharacteristics of the relationships have a positively reduce risky behavior and good health outcomes of emerging adult (Helgeson, Palladino, Reynolds, Becker, Escobar \& Siminerio, 2013). Peers of emerging adults with long term illnesses are important because they can buffer, exacerbate the illness impact, and help the individual feel accepted (Scholes,
Mandleco et al., 2012). In that sense, emerging adults need diabetic specific support within their relationships with family, friends, and romantic partners that serves as resources within their diabetic management (Hanna 2012). Relationships on the basis of acceptance of the chronic illness can increase cooperation with the regime of diabetes glucose checks, insulin injections, and adherence to seeking the help of health care professionals, which pose many benefits to the emerging adult. Within parental relationships, open communication and parental acceptance facilitate the development of autonomy which encourages ownership of one's experiences. Peer relationships that are accepting of the illness will allow the individual with the illness to not fear consequences of accommodation, feeling "different," or peer conflict/negligence (Scholes, Mandleco et al 2012).

A change within supportive network may add to the emotional distress experienced. Within this period, it is supported that parental involvement decreases and peer support is lower in emerging adults with diabetes (Hanna 2012). As emerging adults "require support from significant others to maintain health and high quality of life," an interruption in the supportive network may cause hindrance in diabetic care-friend conflict and low parental involvement resulted in poorer glycemic control (Monaghan, Helgeson et al. 2015). In some case, lack of parental/peer support may cause the emerging adult to become careless in diabetes management to fill the deficit of attention (Pyatak 2011).

\section{Delivery of Diabetes Care:}

Young adults with diabetes face an additional challenge: the transition of their diabetes health care services from the pediatric care team to the adult health care team (Fleming, Carter et al. 2002). The transition from pediatric to adult care settings represents a formidable change in healthcare delivery for adolescents and emerging adults with chronic illness, and may be particularly challenging for those with Type I DM, an illness requiring a high level of patient involvement in selfcare (Monaghan, Helgeson et al. 2015). The transition from pediatric to adult health care really is a passage between two care subcultures situated in different contexts within the overall health care context (Lundin 2008).

Pediatric and adult health care systems place different demands and expectations on individuals with diabetes (Monaghan, Helgeson et al. 2015). The approach to children's health care places far greater emphasis on the family. The adult health care system is much more individually directive and impersonal. Provider-patient interactions in adult care discourage the youth from having parents present. It is often taken for granted that the patient has the necessary cognitive skills to manage their own care (Fleming, Carter et al. 2002). Diabetes self-management education is a critical element of care for all people with diabetes and is necessary in order to improve diabetes outcomes (Funnell, Brown et al. 2011). Most diabetes educational materials focus on children or adults, but not young adults, who have distinct needs (Safaii, Raidl et al. 2013). Anderson and Wolpert (2004) state that diabetes education and care during the young adult period need to be framed in the context of complex individual changes: cognitive, social, emotional, educational, and familial.

Young people must make a difficult transition involving a sudden move between two very different healthcare 
systems. While coping with the pressures of the transition, emerging young adults are expected to have the time and motivation to keep their diabetes under control. Perhaps, then, it is not surprising that non-adherence is an issue among adolescents with diabetes (Fleming, Carter et al. 2002). This increases the risk for gaps in diabetes care during the transition between pediatrics and adult health care (Garvey, Wolpert et al. 2012). Such gaps contribute to poorer glucose control, missed medications, and ultimately more risks for disease progression.

\section{Discussion:}

Young adulthood has been identified as a period of critical vulnerability to negative health outcomes for individuals with diabetes. Young adults are particularly apt to engage in exploratory and risk-taking behaviors that are often incompatible with the high degree of responsibility needed to manage such a complex disorder (Pyatak 2010). In response to a world pandemic of diabetes, most of diabetes programs focus on Type II Diabetes Mellitus (Type II DM) or children with Type I DM. This focus misses the transitional group of young adults with Type I DM with special demands (Mbanya 2011). Individuals in this vulnerable population experience significant disparities in both the processes of care and health outcomes relevant to diabetes management (Borak, SalipanteZaidel et al. 2012, DoostiIrani, Abdoli et al. 2013). These individuals fall outside the focus of the current US health care system with the typical agendas of either pediatric or adult care only (Weissberg-Benchell, Wolpert et al. 2007). They are faced with the burden of searching for and accessing resources for diabetes management, which is a struggle that overwhelms most adults, let alone young adults in transition (Lewis 2011). They experience poor glycemic control, poor quality of life, poor psychosocial wellbeing, discrimination and stigmatization with a high morbidity and mortality rates.

Ironically, we have emphasized the vulnerability of youth with diabetes. Yet adolescence and young adulthood are usually marked by feelings of invulnerability and a tendency to resist adult control, which may further limit receptiveness to recommendations for diabetes treatment (Peters, Laffel et al. 2011). This indicates an urgent need for effective treatment strategies to improve metabolic status in youth with diabetes (Petitti, Klingensmith et al. 2009). The needs of emerging adults with diabetes require a multifaceted approach (Weissberg-Benchell, Wolpert et al. 2007). However, there is limited information on how best to provide developmentally appropriate care to young adults with diabetes (Pyatak 2010).

A gap currently exists between the promise and the reality of diabetes care. Further research is needed to develop evidence-based interventions specifically for young adults. Practical interventions that facilitate collaborative relationships and foster patient-centered practices are the key to closing this gap (Funnell and Anderson 2004). Research focused on examining individuals with Type I DM, parent, and provider attitudes and behaviors, as well as health care system structural processes and obstacles will help to identify potential organizational causes and interventions that may mitigate the risk of deteriorating metabolic control in this population. Young adults who experience transition to adult care require additional support to maximize their health outcomes (Lotstein, Seid et al. 2013). Increase in the absolute numbers of youth with diabetes in this transition period highlight the need for a framework of care and education for this population and the necessity of additional research in this area. The next decade should see the emergence of evidencebased strategies that support best practice for the growing numbers of young adults with Type I DM (Peters, Laffel et al. 2011). That this is an unjust health disparity, and that the system is exclusionary by not meeting young adults where they are. And the division in care between peds and adult are really just modeled by medical specialty, --not patientcentered, as PCORI demands.

\section{Disclaimers:}

This study has not received any funding and authors do not have any conflict of interest.

\section{References:}

Abdoli, S., Ashktorab, T., Ahmadi, F., Parvizy, S., \& Dunning, T. (2014). Seeking new identity empowerment; stories of Iranians living with diabetes. Iranian Journal of Nursing and Midwifery Research; 19(2): 145-151.

Anderson, B. J., and Wolpert, H.A. (2004). A developmental perspective on the challenges of diabetes education and care during the young adult period. Patient Education and Counseling; 53: 347-352.

Borak, J., Salipante-Zaidel, C., Slade, M. D. \& Fields, C.A. (2012). Mortality Disparities in Appalachia Reassessment of Major Risk Factors. JOEM; 54(2): 146-156.

DoostiIrani, M., Abdoli, S., Parvizi, S., Fatemi, N.S., \& Amini, A. (2013). Living in the shadow and light: Iranian youths' responses to diabetes-related stigma. European Online Journal of Natural and Social Sciences; 2(2): 439-448.

Dovey-Pearce, G., Doherty, Y., \& May, C. (2007). The influence of diabetes upon adolescent and young adult development: a qualitative study. Br J Health Psychol; 12(1): 75-91.

Fleming, E., Carter, B., \& Gilibrand, W. (2002). The transition of adolescents with diabetes from the children's health care service into the adult health care service: a review of the literature. Journal of Clinical Nursing; 11: 560-567.

Garvey, K. C., Wolpert, H.A., Rhodes, E.T., Laffel, L.M., Kleinman, K., Beste, M.G. et al. (2012). Health care transition in patients with type 1 diabetes young adult experiences and relationship to glycemic control. Diabetes Care; 38(12): 1716-1722.

Gill, G., Nayak, A.U., Wilkins, J., Hankey, J., Raffeeq, P., Varughese, G.I., et al. (2014). Challenges of emerging adulthood-

transition from pediatric to adult diabetes. World J Diabetes; 5(5): 630-635.

Hanlan, M. E., Griffith, J., Patel, N., \& Jaser, S.S. (2015). Eating disorders and disordered eating in type 1 diabetes: prevalence, screening, and treatment options. Curr Diab Rep.HHS (2014). National Prevention Strategy- ELIMINATION of HEALTH DISPARITIES. Department of Health and Human Service. 
Johnson, B., Eiser, C., Young, V., Brierley, S., \& Heller, S. (2013). Prevalence of depression among young people with Type 1 diabetes: a systematic review. Diabet Med; 30(2): 199-208.

Kipps, S., Bahu, T., Ong, K., Ackland, F.M., Brown, R.S., Fox, C.T., et al. (2002). Current methods of transfer of young people with Type 1 diabetes to adult services. Diabetic Medicine; 19(8): 649-654.

Lewis, V. A. (2011). The Challenges of diabetes management for emerging young adults. Diabetes Spectrum; 24(1): 4-5.

Lotstein, D. S., Seid, M., Klingensmith, G., Case, D., Lawrence, J.M., Pihoker, C., et al. (2013). Transition from pediatric to adult care for youth diagnosed with type 1 diabetes in adolescence. Pediatrics; 131(4).

Lundin, C. S. (2008). Living with diabetes during transition to adult life- relationships, support of selfmanagement, diabetes control and diabetes care. University of Gothenburg, Sahlgrenska Academy.

Luyckx, K., Rassart, J., Aujoulat, I., Goubert, L., \& Weets, I. (2016). Self-esteem and illness self-concept in emerging adults with type 1 diabetes: Long-term associations with problem areas in diabetes. Journal of Health Psychology; 21(4): 540-549.

Luyckx, K., Seiffge-Krenke, I., Schwartz, S. J., Goossens, L., Weets, I., Hendrieckx, C., et al. (2008). Identity development, coping, and adjustment in emerging adults with a chronic illness: The sample case of type 1 diabetes. Journal of Adolescent Health 43.

Mbanya, J. C. (2011). A very special issue in a stellar year for diabetes. Diabetes Voice 56(2): 4.

Melton, S.T., and Johnson, L.N. (2015). Using photoelicitation to explore young adult perspectives on coping with diabetes. The Plaid journal; 1(1): 4048.
Monaghan, M., Helgeson, V., \& Wiebe, D. (2015). Type 1 diabetes in young adulthood. Curr Diabetes Rev; 11(4): 239-250.

Palladino, D. K., Helgeson, V.S., Reynolds, K.A., Becker, D.J., Siminerio, L.M., \& Escobar, O. (2013). Emerging adults with type 1 diabetes; A comparison to peers without diabetes. J Pediatr Psychol;38(5): 506-517.

Peters, A., Laffel L., \& the American Diabetes Association Transitions Working Group (2011). American Diabetes Association Transitions Working Group Diabetes care for emerging adults: recommendations for transition from pediatric to adult diabetes care systems. Diabetes Care 34: 2477-2485

Petitti, D. B., Klingensmith, G.J., Bell, R.A., Andrews, J.S., Dabelea, D., Imperatore, S. et al. (2009). Glycemic control in youth with diabetes: The SEARCH for diabetes in youth study. J Pediatr; 155(5): 668-672: e661-663.

Pyatak, E. A. (2010). Diabetes management in the everyday lives of emerging adults. University of Southern California.

Safaii, S., Raidl, M., \& Ramsay, S. (2013). Young adults with diabetes discuss barriers and solutions to diabetes management. Advances in Diabetes and Metabolism; 1(1): 6-11.

Weissberg-Benchell, J., Wolpert, H., \& Anderson, B.(2007). Transitioning from pediatric to adult care: a new approach to the post-adolescent young person with type 1 diabetes. Diabetes Care; 30: 2441-2446

White, R. O., Beech, B.M., \& Miller, S.(2009). Health care disparities and diabetes care: Practical considerations for primary care providers. Clinical Diabetes; 27(3): 105-112. 